\title{
El aprendizaje integrado de la lengua española y los contenidos de áreas no lingüísticas en los proyectos lingüísticos de centro
}

\author{
Alejandro Gómez Camacho \\ Universidad de Sevilla
}

Recibido: 3 mayo 2012 / Aceptado: 1 diciembre 2012

ISSN: $1697-7467$

\begin{abstract}
RESUMEN: La generalización de los proyectos lingüísticos de centro implica una profunda revisión en la práctica didáctica en la lengua de instrucción para adecuarla a la nueva realidad de los programas de bilingüismo.

Este artículo ofrece una propuesta concreta para incorporar la lengua española a la metodología que integra el aprendizaje de contenidos no lingüísticos y lenguas; en ámbitos como la ortografía, el trabajo sobre los géneros textuales y la comprensión lectora de textos de las áreas no lingüísticas.

La propuesta trasciende el ámbito de la educación bilingüe para extenderse a todo el alumnado como medida de atención a la diversidad.

Palabras clave: Currículo integrado, lengua española, PLC.

Integrated Learning of Spanish and the Content of Non-Linguistic Areas in School Language Projects

ABSTRACT: The development of the School Language Projects implies a thorough review of the teaching practice in the L1s in order to adapt them to bilingual education.

A specific proposal is offered in this paper to work content and language integrated learning methodology in Spanish. It focuses on areas such as spelling, text genres and reading comprehension of texts from non-linguistic areas.

Furthermore, this research goes beyond bilingual teaching since it is also used as a tool to cope with attention to diversity measures.
\end{abstract}

Keywords: Integrated curriculum, Spanish, SLP.

\section{INTRODUCCIÓN}

El proyecto lingüístico de centro es un plan integral para el desarrollo de la competencia lingüística en los centros de enseñanza (AA.VV., 2011). Los PLC constituyen una oportunidad en estos momentos de cambio del sistema educativo español para el profesorado de lengua castellana y literatura; tanto para modernizar la práctica didáctica en la lengua española, como para realizar una aportación sustancial al PLC desde los departamentos didácticos. 
En la normativa que regula la enseñanza bilingüe en nuestro entorno, aparece por primera vez la obligación de desarrollar un currículo integrado de las lenguas y materiales para el aprendizaje integrado de contenidos y lenguas extranjeras en el marco del proyecto educativo de cada centro; así como el uso del Porfolio Europeo de las Lenguas. En el mismo sentido, se impone el establecimiento de vínculos entre las distintas lenguas, unificar la terminología lingüística y los planteamientos metodológicos basados en un enfoque comunicativo.

A pesar de que el aprendizaje integrado de contenidos y lenguas se asocia normalmente con la adquisición de la competencia lingüística en lengua extranjera, no hay duda de que la lengua de instrucción forma parte de la educación bilingüe, una obviedad que no siempre se reconoce en los centros educativos por parte del profesorado de lengua castellana y literatura. Gómez Vidal (2007), Pavón (2011), Madrid (2011), y Lorenzo, Trujillo y Vez (2011), entre otros, analizan las repercusiones positivas de la implantación de las secciones bilingües en España, tanto en las escuelas de educación primaria como en los institutos de enseñanza secundaria. Pérez y Zayas (2007: 221), se preguntan expresamente por el motivo de que la enseñanza AICLE goce de un reconocimiento generalizado en nuestro país aplicado al aprendizaje de lenguas extranjeras, mientras que el aprendizaje de contenidos de todas las áreas del currículo apenas contribuye al dominio de las lenguas de instrucción, en nuestro caso la lengua española, aunque también se use para aprender. Trujillo (2007: 76) recuerda que en nuestra tradición didáctica la lengua española se aleja de los programas basados en tareas para perpetuar los programas formales en los que la expresión oral y escrita y el texto como objeto de creación y estudio están todavía ausentes de gran parte de las aulas. La relación entre los programas bilingües y la competencia en lengua materna está ampliamente estudiada en nuestro contexto (Madrid y Hughes, 2011: 135 y sig.), tanto en la educación primaria como en la secundaria. Este artículo pretende hacer una propuesta concreta y contextualizada para que el profesorado de lengua castellana y literatura aproveche la experiencia del aprendizaje integrado de contenidos y lengua extranjera para modernizar definitivamente la práctica didáctica de la lengua de instrucción, integrando la competencia lingüística en la lengua materna en los proyectos lingüísticos de centro.

La inserción definitiva de la lengua castellana y la literatura en la metodología de la educación bilingüe implica la superación del enfoque centrado en contenidos gramaticales e historia de la literatura para incorporar el trabajo por competencias que consagra el currículo vigente para la educación obligatoria (primaria y secundaria obligatoria), en la certeza de que la competencia básica en comunicación lingüística en la lengua de escolarización (Navarro, 2010), incluso para hablantes nativos, tiene su principal instrumento en los proyectos lingüísticos de centro, la integración de los currículos de las lenguas y el aprendizaje integrado con las mal llamadas áreas no lingüísticas. No entraremos aquí en las implicaciones metodológicas que tendría para el aula de lengua castellana y literatura la integración con las áreas no lingüísticas y las otras lenguas del currículo ni en la excesiva dependencia de la tradición lingüística y filológica (Bernárdez, 2008); pero baste con apuntar, como recuerda Lorenzo (2010), que la asunción de un PLC implica que el texto en sus diferentes géneros (y no la oración) sería necesariamente la unidad lingüística básica para todas las lenguas; el trabajo sistemático sobre los diferentes géneros textuales compartidos con el resto de las materias del currículo en un enfoque comunicativo obliga a que los contenidos gramaticales vinculados al análisis oracional no sean un fin en sí mismos, sino un instrumento al servicio de la adquisición de la competencia lingüística que caracteriza a los hablantes cultos en las 
diferentes etapas educativas. Los PLC son sin duda un eficaz instrumento para superar la excesiva gramaticalización del currículo de la L1 que criticaba Trujillo (2007).

Al profesorado de lengua española y de las otras lenguas presentes en el proceso de enseñanza aprendizaje corresponde la elaboración del currículo integrado de las lenguas y participar en la elaboración de materiales AICLE junto al profesorado de áreas no lingüísticas; dos elementos esenciales del PLC de un centro bilingüe que pueden extenderse al proyecto educativo de cualquier centro que conceda a la competencia en comunicación lingüística un lugar destacado entre los objetivos que pretende priorizar. Los reglamentos de organización de centros aportan como novedad en secundaria el agrupamiento de los departamentos por áreas de competencias; de esta manera, todas las áreas lingüísticas comparten dentro del área de competencia social-lingüística la obligación de que sus programaciones participen de una visión integrada y multidisciplinar de sus contenidos, así como favorecer el trabajo en equipo del profesorado perteneciente al área para el desarrollo de las programaciones didácticas.

El currículo integrado de las lenguas y el aprendizaje integrado de contenidos y lenguas extranjeras, junto a la atención a la diversidad y los planes de mejora, conforman, en opinión de Fernando Trujillo (AA.VV., 2011: 8-9), los elementos constitutivos de los PLC.

Como se ha apuntado antes, la elaboración de un proyecto lingüístico de centro como parte esencial del proyecto educativo no debe asociarse en exclusiva a los centros con enseñanza bilingüe; la elaboración de un currículo integrado de las lenguas que se estudian en el aula, así como de medidas que integren la enseñanza de las lenguas (por supuesto de la lengua española) en los contenidos de otras materias, permiten la mejora de la competencia lingüística en centros que no han incluido el bilingüismo en su proyecto educativo. Una de las medidas de atención a la diversidad que se recogen con más frecuencia en la normativa vigente es precisamente la integración de las diferentes materias en ámbitos (por ejemplo, en los programas de diversificación curricular y en los programas de cualificación profesional inicial), consecuentemente la integración del currículo que desarrolla el aprendizaje de la lengua castellana y la literatura con el de las otras lenguas presentes en las aulas, y la vinculación del desarrollo de la competencia lingüística en español a las mal llamadas áreas no lingüísticas, constituye un instrumento inexcusable para la atención a la diversidad y para el aprendizaje de la L1 en cualquier centro. En el mismo sentido, Ganga (2011), y Cassany, Esteve y Pérez-Vidal (2009) destacan el papel del Porfolio Europeo de las Lenguas como una herramienta adecuada para favorecer la reflexión de los alumnos con dificultades de aprendizaje de la lengua extranjera en programas de diversificación curricular.

\section{Los PROYECTOS LINGÜÍSTICOS DE CENTRO}

Por su propia naturaleza, un plan integral para el desarrollo de una de las competencias básicas solo puede generarse dentro del proyecto educativo de cada centro donde se fijan los valores, los objetivos y las prioridades de actuación que se establecen como expresión del compromiso del centro en la comunidad en la que está inserto. La concreción de estos objetivos propios, dentro de los que estableciere la legislación vigente en cada caso, marcará la diferencia entre los PLC de un centro que aspira a ser bilingüe en español e inglés, un centro de educación compensatoria que se propone como meta la integración de la lengua española como L1 en áreas del currículo que resulten atractivas al alumnado, centros 
con una fuerte presencia de alumnado cuya lengua materna no coincide con la lengua de escolarización o centros que se insertan en comunidades bilingües dentro de España. No hay que identificar por tanto el PLC con centros en cuyo proyecto educativo se establece como una seña de identidad convertir a su alumnado en hablantes competentes de lenguas europeas de prestigio.

La asunción de un PLC implica el compromiso de toda la comunidad educativa en la adquisición de la competencia lingüística. En las comunidades autónomas con lengua propia se destaca la adquisición de la competencia lingüística en la lengua de escolarización en una tradición que se remonta a finales del pasado siglo XX; baste recordar el monográfico que la revista Textos dedicaba al proyecto lingüístico de centro concebido como un instrumento para las comunidades autónomas en las que el alumnado es efectivamente bilingüe. La estructura de un PLC parece sujeta a las necesidades educativas del centro que lo diseña. Tanto en el informe de la comisión que estudió los PLC en Cataluña como en las orientaciones para la elaboración de los PLC que se han publicado en Andalucía, por citar dos ejemplos, se ofrecen modelos adecuados a la realidad de cada comunidad.

Un proyecto que pretende la mejora de la competencia comunicativa en un centro implica a la práctica totalidad del proceso de enseñanza aprendizaje, desde la programación de aula hasta la elaboración del proyecto educativo del centro. Si revisamos brevemente la estructura organizativa de la enseñanza en la educación obligatoria, llegaremos a la conclusión de que los PLC efectivamente son una apuesta muy ambiciosa de toda la comunidad educativa que compromete al profesorado en su conjunto.

En lo que se refiere a los proyectos educativos de cada centro, la puesta en práctica de un PLC afectaría, al menos, a estos aspectos:

- Objetivos propios para la mejora del rendimiento escolar

- Líneas de actuación pedagógica

- Coordinación y concreción de los contenidos curriculares

- Los procedimientos y criterios de evaluación

- La forma de atención a la diversidad del alumnado

- Los criterios para organizar y distribuir el tiempo escolar

- Los procedimientos de evaluación interna

- Los criterios para establecer los agrupamientos del alumnado

Del mismo modo, las áreas de competencias de los centros de enseñanza secundaria se verían sustancialmente implicadas en sus acuerdos por el PLC, pues por su propia naturaleza tienen entre sus funciones la coordinación de las actuaciones para que las programaciones didácticas proporcionen una visión integrada y multidisciplinar de sus contenidos, la utilización de métodos pedagógicos y proponer actividades que contribuyan a la adquisición por el alumnado de las competencias asignadas a cada área (en este caso, la lingüística). El diseño de un PLC obligará sin duda a reforzar el trabajo en equipo del profesorado para el desarrollo de las programaciones didácticas que se insertan en el área de competencias.

Las programaciones didácticas (elaboradas por los departamentos de coordinación didáctica o por los equipos de ciclo) constituyen la aportación más significativa a los PLC, sin olvidar el último nivel de concreción de las mismas que serían las programaciones de aula (Guillén, 2009); inexcusablemente algunos elementos de las programaciones habrían de adecuarse a los acuerdos que implica un PLC: 
- Los objetivos, los contenidos y su distribución temporal, y los criterios de evaluación

- La contribución de la materia a la adquisición de las competencias básicas

- La metodología

- Los procedimientos de evaluación y los criterios de calificación

- Las medidas de atención a la diversidad

- Los materiales y recursos didácticos que se vayan a utilizar

- Las actividades complementarias y extraescolares relacionadas con el currículo

Pero no solo hay que tener en cuenta la estructura que abarca desde el proyecto educativo de un centro hasta la programación de secuencias didácticas de aula para cada grupo; el PLC también condicionaría sustancialmente el trabajo de los equipos docentes (llevan a cabo el seguimiento global del alumnado del grupo, estableciendo las medidas necesarias para mejorar su aprendizaje), del departamento o equipo de orientación (asesora en las medidas de atención a la diversidad) y del equipo técnico de coordinación pedagógica (establecen las líneas generales de actuación pedagógica), y del equipo directivo.

El nuevo departamento de formación, evaluación e innovación educativa de los institutos de secundaria también estaría directamente implicado por el diseño y la puesta en práctica de un PLC (investiga sobre el uso de las buenas prácticas docentes, fomenta el trabajo cooperativo de los equipos docentes y vela para que estos contribuyan al desarrollo de las competencias básicas, y fomenta iniciativas entre los departamentos de coordinación didáctica que favorezcan la elaboración de materiales curriculares).

\section{LA INTEgRaCión de LA LENGUA ESPAÑola EN CONTENidos de Otras ÁREAS}

Los PLC condicionan a todo el profesorado y a toda la organización del centro, desde los equipos educativos hasta los órganos de coordinación pedagógica; desde la puesta en práctica de secuencias didácticas para el aula hasta la estructura misma del proyecto educativo; sin embargo, los PLC solo tienen posibilidades de éxito si la competencia comunicativa en lengua española (la lengua de escolarización en nuestro entorno) se constituye en un elemento esencial del mismo. En la práctica, las secuencias didácticas para el aprendizaje integrado de contenidos y lengua extranjera dan por supuesta la competencia en lengua española y se centran en la adquisición de habilidades comunicativas en la L2 y la L3; lo que con frecuencia tiene como resultado una escasa implicación del profesorado de lengua española en los programas bilingües y, como consecuencia, en los PLC. El profesorado de lengua castellana y literatura debe ser consciente de que el modelo de integración de contenidos (de áreas no lingüísticas) y lenguas es una buena idea también para la lengua española (Sierra y Lasagabaster, 2008); el hecho de que se trabaje con éxito la competencia comunicativa en lenguas extranjeras en otras áreas solo puede llevarnos a la conclusión de que la lengua de escolarización debe aprovechar ese modelo.

En ningún caso se pretende que el profesorado de las áreas no lingüísticas se encargue de enseñar lengua centrada en contenidos gramaticales (Pavón, 2011) ni deben insertarse estos contenidos en las secuencias didácticas ni en las programaciones de estas materias; por 
el contrario, se trata de trabajar las competencias en comunicación lingüística (expresión y comprensión, oral y escrita) en hablantes más o menos competentes que están adquiriendo nuevo vocabulario y cuyas necesidades comunicativas se incrementan en el proceso de enseñanza aprendizaje. En otras palabras, se trata de aprender el lenguaje de las matemáticas, de las ciencias naturales, de las actividades deportivas, etc. (Lorenzo, Trujillo y Vez 2011); el objetivo último de muchas de las áreas no lingüísticas implica que el alumnado incorpore un vocabulario específico nuevo como vocabulario activo o de expresión en textos coherentes y cohesionados que se adecuen a las diferentes situaciones de comunicación.

Para el profesorado de lengua castellana y literatura será todo un reto que el aprendizaje integrado de contenidos de las áreas no lingüísticas y las lenguas extranjeras se extienda también a la lengua de escolarización, siguiendo el modelo que se utiliza para las lenguas extranjeras. El problema, como indica Vollmer (2007:15), estaría en cómo se desarrollan efectivamente estas competencias (incluida la comunicación lingüística en lengua materna) en las áreas no lingüísticas.

Desde el punto de vista epistemológico queda clara la necesidad de integrar el área de lengua castellana y literatura en los proyectos lingüísticos de centro a través de la metodología del aprendizaje integrado con los contenidos de las áreas no lingüísticas. Es el momento de formular propuestas concretas desde la experiencia de la práctica docente en el aula de lengua española: propondremos algunos ejemplos en los que la integración en el PLC de contenidos que tradicionalmente se asociaban al área de lengua castellana y literatura tendría como consecuencia la formación de hablantes competentes en nuestra lengua.

Quizá la aportación más evidente del área de lengua castellana y literatura al desarrollo de la competencia lingüística en las áreas no lingüísticas (ciencias sociales y áreas de competencias científico-tecnológica y artística) estaría en el ámbito de la expresión escrita. A modo de ejemplo, nos referiremos a la ortografía. Con frecuencia la escasa competencia ortográfica del alumnado se relaciona con un planteamiento didáctico equivocado: la identificación de la ortografía como parte de la gramática, como un conjunto de reglas que permiten escribir sin faltas; en otras palabras, la identificación de la escritura correcta (ortografía) con las asignaturas de lengua castellana y literatura (Gómez Camacho, 2009).

Sin embargo, la ortografía no es parte de la gramática, sino de la expresión escrita, y tiene mucha más relación con la adquisición del vocabulario activo o de expresión que con ninguna regla. En este contexto, la adquisición de la competencia ortográfica asociada exclusivamente a la asignatura de lengua castellana y literatura está abocada al fracaso; escribir correctamente es una característica de los hablantes competentes que utilizan el español para comunicarse y atañe por igual a cualquier actividad en la que se escriba.

Los ejercicios tradicionales relacionados con la ortografía son de muy dudosa eficacia, los recursos didácticos (ejercitación directa del vocabulario ortográfico, ejercicios de atención, de pronunciación ortológica, memorización, evocación y aplicación de reglas, compleción de textos, etc.) deberían seleccionarse en función de las características lingüísticas del hablante, entendiendo que el objetivo es la automatización de la norma ortográfica; el objetivo es escribir correctamente, no saber ortografía (Gómez Camacho, 2007). Cualquier ejercicio que no esté directamente relacionado con la producción de textos reales de comunicación carece de utilidad; en este sentido, la adquisición de una verdadera competencia ortográfica en lengua española (sobre todo para hablantes nativos competentes que presentan dificultad en un vocabulario expresivo concreto) solo es posible desde un enfoque integrado en el aprendizaje y la producción de textos en áreas no lingüísticas. 
La elaboración de listas de palabras con el vocabulario que cada hablante necesita escribir según sus necesidades comunicativas; pero cuya ortografía no ha automatizado (inventarios cacográficos individuales o de aula), es un recurso didáctico muy eficaz que comparten todas las áreas del currículo. Como indica Gómez Camacho (2008), el inventario cacográfico es un recurso didáctico adecuado para los hablantes competentes que persisten en errores ortográficos en su vocabulario expresivo escrito. Se trata de un pequeño vocabulario en el que cada hablante anota las palabras en las que ha tenido dudas o ha cometido errores al escribir. Evidentemente es una lista abierta; conforme se amplía el vocabulario expresivo, aumenta el número de palabras en las que aparecen dudas o errores ortográficos. Implica al propio alumno, que se convierte en el verdadero protagonista del aprendizaje, al definir su vocabulario expresivo escrito (las palabras que realmente necesita escribir) y al confirmar el origen de cada error en su caso personal (desatención, hablas dialectales, vocabulario expresivo insuficiente, desconocimiento de reglas, etc.).

El equipo educativo (todos los profesores de todas las áreas) comparte un mismo recurso didáctico que se recoge en el PLC, lo que permite que la expresión escrita y la ortografía sean un verdadero objetivo general de la etapa. Los inventarios cacográficos no necesitan conocimientos lingüísticos ni filológicos previos, tan solo la reflexión ortográfica de cada hablante y el deseo de escribir sin faltas. El equipo educativo en su conjunto, en el contexto del PLC, desplaza al profesorado de lengua como único agente en el proceso de enseñanza aprendizaje de la competencia comunicativa escrita. A fin de cuentas, saber una materia no es más que utilizar el vocabulario preciso en textos adecuados y aceptables, y saber una palabra implica necesariamente ser capaz de escribirla conforme a la norma culta establecida (Cassany, Luna y Sanz, 1994). Con respecto a la evaluación, se supera el tradicional recuento de faltas en un texto para evaluar la adquisición del vocabulario expresivo más frecuente en cada hablante; ya no se trata de cuantificar cuántas palabras se escriben mal, sino de que el propio alumno sea consciente de cuántas palabras importantes ha aprendido a escribir correctamente.

En conclusión, los inventarios cacográficos son un excelente instrumento que el área de lengua castellana y literatura puede aportar al PLC, ofreciendo un nuevo planteamiento muy eficaz para la adquisición de la competencia ortográfica totalmente desligado de la tradición que asocia ortografía a lengua española. La repetición de modelos didácticos diseñados para quienes están aprendiendo la expresión escrita o la realización directa de los ejercicios de ortografía están destinadas al fracaso; el contacto con los textos escritos sin un objetivo claro y sin una reflexión ortográfica previa (la idea de que para mejorar la ortografía basta con leer mucho) tampoco es efectivo para usuarios competentes, la inclusión en el PLC de esta metodología, como parte del proyecto educativo del centro, es sin duda una propuesta mucho más eficaz.

Otra de las aportaciones concretas que puede realizar el profesorado de la lengua de instrucción al PLC sería la propuesta de una clasificación clara y contextualizada en el centro de los textos que el alumnado utiliza para desarrollar su competencia lingüística, así como las características textuales que permiten su explotación didáctica en el proceso de enseñanza aprendizaje. En muchas ocasiones, las dificultades en la integración de los currículos de las áreas lingüísticas y no lingüísticas proceden de una terminología contradictoria sobre los textos y las competencias comunicativas que se ejercitan en el aula.

Puesto que todas las programaciones didácticas deben incluir actividades relacionadas con la lectura, la escritura y la comunicación oral, el diseño coordinado de estas actividades 
para la producción y la utilización didáctica de determinadas tipologías textuales sería el punto de partida más adecuado para elaborar un PLC. Se trataría de llegar a un acuerdo sobre las tipologías textuales que se utilizarían (textos narrativos, descriptivos, instructivos...; de los medios de comunicación, académicos, divulgativos, literarios, electrónicos...), los temas sobre los que se hablaría, leería o escribiría (por ejemplo, los temas transversales, la actualidad, temas de interés para el alumnado) y la forma de integrar las diferentes materias (proyectos, CIL, AICLE...); tal y como sugiere la comisión que estudió el PLC en Cataluña (Junyent, 2007).

La dificultad reside muchas veces en tipologías textuales que se dan por supuestas sin que haya acuerdo sobre las mismas. Por ejemplo, definir o demostrar son actividades que generan textos en todas las áreas del currículo; pero no son fáciles de sistematizar en el contexto de un centro educativo. Igualmente, los hablantes competentes que cursan la enseñanza obligatoria utilizan textos reales de comunicación que satisfacen sus necesidades comunicativas fuera del aula; pero la explotación didáctica de los twits, de los posts de un blog, o las entradas que se escriben en el espacio personal o en el tablón de Tuenti (o una biografía en Facebook) implican un esfuerzo de reflexión sobre estas nuevas tipologías textuales de la comunicación electrónica que con demasiada frecuencia quedan fuera del aula.

En un estudio ya clásico, (Jorba, Gómez y Prat, 2000: 31-32) se clasifican siete habilidades cognitivolingüísticas que originan los textos que se usan con más frecuencia en la escuela: describir, definir, resumir, explicar, justificar, argumentar y demostrar.

¿Cuáles son las habilidades cognitivas que se activan al producir un texto descriptivo, un texto narrativo, un texto explicativo, un texto argumentativo o un texto instructivo? Esta clase de habilidades las denominaremos cognitivolingüísticas porque están estrechamente relacionadas con las tipologías textuales (...). Podemos incluir en esta categoría habilidades como: describir, definir, resumir, explicar, justificar, argumentar y demostrar. Habilidades que, si bien son transversales, se concretan de manera diferenciada en cada una de las áreas curriculares. En consecuencia, no es un aspecto que deba ser abordado sólo desde el área de lengua sino que debe afrontarse desde las diversas áreas curriculares si no se quiere caer en la trampa de producir textos cuya estructura esté de acuerdo con las características marcadas desde las tipologías textuales, pero vacíos de contenido.

No vamos a entrar ahora en el análisis de las diferentes habilidades cognitivolingüisticas ni en la comparación entre las diferentes tipologías textuales que las reclaman; pero no cabe duda de que un PLC que unifique qué se entiende por demostrar, definir o resumir, para todos los textos que genere el alumnado en todas las áreas durante toda la etapa educativa, constituirá un instrumento pedagógico sumamente útil. Y como evidentemente una demostración (por citar un ejemplo) implica diferentes matices en matemáticas, ciencias naturales o ciencias sociales, puede ser el área de lengua castellana y literatura el punto de partida que unifique los diferentes puntos de vista en una estrategia didáctica común y coordinada. Se trataría de diagnosticar qué tipos de textos generan los alumnos para satisfacer sus necesidades comunicativas reales (dentro y fuera del aula), y proponer una caracterización de los mismos compartida por toda la comunidad educativa, en la línea que proponen Madrid y Hughes (2011: 202) sobre la aportación del área de las ciencias sociales a la competencia 
El aprendizaje integrado de la lengua española y los contenidos...

comunicativa, tanto para estudiantes monolingües como para estudiantes de programas de bilingüismo.

En la misma línea, otro de los aspectos fundamentales en el que puede participar el área de lengua castellana y literatura al PLC sería el diseño de tareas comunicativas conjuntas con las áreas no lingüísticas (en las Orientaciones metodológicas para la elaboración y puesta en marcha de un proyecto lingüístico en los centros de la Comunidad Autónoma de Andalucía se sugiere al menos una por trimestre en cada asignatura) partiendo de las diferentes tipologías textuales según la materia (definiciones, demostraciones, explicaciones, instrucciones...) que desarrollen destrezas comunicativas en español (escuchar, hablar, leer y escribir).

El modelo que aportan las lenguas extranjeras L2 y L3 para la intervención en el aprendizaje integrado de contenidos y leguas extranjeras AICLE en los centros bilingües es un referente válido para la lengua de escolarización o L1. En este sentido, Pérez Esteve y Zayas (2007: 220-221), insisten en que la inclusión de la competencia básica en comunicación lingüística en la lengua de instrucción (la lengua española en nuestro caso) debe seguir el modelo de las estrategias pensadas para la lengua extranjera, en especial el aprendizaje integrado de contenidos y lenguas. Lorenzo (2010: 404) analiza detalladamente la utilización de los géneros textuales en programas integrados en el currículo en una propuesta que incluye expresamente la lengua materna.

Un ejemplo paradigmático sería la preparación y exposición ante la clase de un texto argumentativo (se trataría de convencer al resto de los compañeros de una tesis, aportando argumentos y rebatiendo las posibles objeciones) sobre un tema relacionado con el área no lingüística; en la secuencia didáctica se incluirían contenidos lingüísticos como por ejemplo realizar presentaciones orales bien estructuradas sobre temas relacionados con la actividad académica o la actualidad que admitan diferentes puntos de vista, utilizando el apoyo de medios audiovisuales y de las tecnologías de la información y la comunicación que aparecen recurrentemente en las programaciones de las asignaturas de lengua castellana y literatura.

En los criterios de evaluación se integrarían criterios lingüísticos como exponer, explicar, argumentar, resumir y comentar, en soporte papel o digital, usando el registro adecuado, organizando las ideas con claridad, enlazando los enunciados en secuencias lineales cohesionadas, respetando las normas gramaticales y ortográficas y valorando la importancia de planificar y revisar el texto. Como procedimientos e instrumentos de evaluación, bastaría con incluir en la coevaluación o autoevaluación del alumnado (o en el registro de observación que se aporte en la secuencia didáctica) ítems sobre la organización y eficacia comunicativa del texto, su planificación, la elaboración de borradores y guiones para la exposición oral, etc. Teresa Ruiz (2008) propone, desde la experiencia que se ha llevado a cabo en el País Vasco para construir una programación conjunta para el tratamiento integrado de las lenguas, diferentes posibilidades para introducir modificaciones en la programación, e insiste expresamente en que el análisis compartido de los criterios de corrección de textos es una de las formas más eficaces de integrar el aprendizaje de contenidos y las lenguas; más allá de la valoración de la ortografía o de los errores sintácticos, propone alcanzar acuerdos para valorar la coherencia de un texto, su adecuación, etc. La utilización del porfolio como instrumento de evaluación compartido por todas las áreas del currículo es otra posibilidad que mejora la actitud y el aprendizaje también en la lengua de instrucción (Cassany, Esteve y Pérez-Vidal, 2009, Ganga, 2011). 
Se trataría por tanto de una secuencia didáctica que probablemente ya se realiza en las materias no lingüísticas (si es que realmente existe alguna que pueda calificarse así) en las áreas de competencias social-lingüística, científico-tecnológica y artística; simplemente se explicitaría en el proceso de enseñanza aprendizaje que la defensa pública de una tesis (de ciencias naturales o sociales, de deportes o arte, de física o matemáticas) es una actividad lingüística que solo tiene sentido si efectivamente se convence, aunque sea parcialmente, a quienes se ha destinado la presentación. Para convencer es importante lo que se dice (contenidos de las áreas no lingüísticas); pero también cómo se dice y en qué orden (competencia lingüística en la lengua utilizada): es el principio básico de la retórica clásica que afecta por igual a cualquier actividad que se realice en el aula o fuera de ella.

La producción oral en el aula no tiene que relacionarse con los textos argumentativos (probablemente los más complejos incluso para hablantes competentes en lengua española) ¿en qué materia no se pide a los alumnos que expliquen, definan, resuman, demuestren, relaten, describan, den instrucciones o expongan?; al hacerlo se generan textos que sirven para progresar en la competencia en comunicación lingüística; sirven para hablar, escuchar, leer y escribir.

Por último nos referiremos a la competencia lingüística más frecuente en el aula: la comprensión lectora en lengua española. En ninguna secuencia didáctica pueden resultar extraños estos contenidos del área de lengua castellana y literatura.

- Comprensión de textos del ámbito académico, atendiendo especialmente a la consulta, en diversos soportes, de diccionarios, glosarios, y otras fuentes de información, incluyendo fragmentos de ensayos

- Utilización de las bibliotecas y de las tecnologías de la información y la comunicación de forma autónoma para la localización, selección y organización de información

- Actitud reflexiva y crítica con respecto a la información disponible ante los mensajes que supongan cualquier tipo de discriminación

También sería fácil asumir como criterio de evaluación inferir el tema general y temas secundarios; distinguir cómo se organiza la información en un texto escrito que se utilice en el aula de cualquier asignatura del área de las ciencias sociales, científico-técnica o de expresión artística.

Normalmente la actividad consistiría en explicar en clase el objetivo de la lectura (para qué se lee el texto, para qué sirve en el contexto de lo que se está aprendiendo, qué información concreta se va a pedir después de la lectura) y su relación con los conocimientos previos del alumnado sobre la información que se pretende que obtenga de la lectura. A continuación, se invitaría a identificar las partes esenciales del texto, cómo se organiza (por ejemplo, con la ayuda de la pizarra digital interactiva); su título, párrafos, apartados, gráficos, ilustraciones, tipografías y cualquier elemento que ayude a jerarquizar la información, a distinguir lo principal de lo accesorio o redundante.

Durante la lectura, se puede sugerir una lectura rápida (un vistazo) con el objeto de encontrar una información precisa o una lectura detallada de todo el texto, se puede inferir el significado de alguna palabra que no se entienda por el contexto (o utilizar el diccionario), subrayar la idea principal de cada párrafo, distinguir ideas generales y específicas, tomar apuntes para elaborar un resumen posterior, etc. Tras la lectura, se podrían planificar 
actividades para aclarar entre los alumnos las dudas que han surgido sobre el texto, elaborar resúmenes o esquemas y compararlos, utilizar la información novedosa para continuar con la tarea propuesta en la secuencia didáctica, releer el texto si se ha comprobado que no se ha entendido suficientemente, etc.

Es evidente que las actividades de comprensión lectora, independientemente del contenido y el tema del texto que se lee, son actividades que implican el desarrollo de la competencia lingüística en todas las áreas del currículo. De nuevo bastaría con explicitar el carácter lingüístico de la lectura en las áreas no lingüísticas e incorporar actividades que lo reconozcan en el diseño de algunas secuencias didácticas que formarían parte del PLC. Una estrategia común para la comprensión lectora en todas las áreas del currículo sería uno de los objetivos fundamentales del PLC en lo referente a la lengua de escolarización, en concreto la lengua española.

\section{Conclusiones}

La generalización de los proyectos lingüísticos de centro como consecuencia de la implantación de los programas de educación bilingüe debe extenderse a todos los centros, independientemente de los objetivos que se prioricen en sus proyectos educativos. Este estudio plantea la necesidad de que la metodología que integra el aprendizaje de lenguas y contenidos no lingüísticos, asociada exclusivamente a las lenguas extranjeras, se aplique a la lengua de instrucción, la lengua española en el caso del sistema educativo andaluz que se toma como referencia.

El enfoque AICLE goza de gran prestigio en nuestro entorno y es reconocido por su eficacia en la adquisición de la competencia lingüística, por lo que su extensión a la lengua de escolarización es, junto a la integración de los currículos de las lenguas presentes en el aula, un instrumento eficaz para la consolidación del trabajo por competencias que consagra el sistema educativo vigente y el MCERL, que sin duda beneficia a la práctica didáctica de la enseñanza de lengua española como lengua de instrucción, en ocasiones demasiado vinculada a la tradición lingüística y filológica propia de otras épocas.

Los proyectos lingüísticos de centro condicionan todo el proceso de enseñanza aprendizaje, desde la elaboración del proyecto educativo a las programaciones didácticas y de aula, desde la selección de objetivos y contenidos a la metodología y la evaluación. Son por tanto el marco adecuado para que la enseñanza de la lengua española como lengua de escolarización actualice sus planteamientos didácticos y los comparta con toda la comunidad educativa.

El trabajo sobre la competencia básica en comunicación lingüística que el currículo prescribe para la enseñanza obligatoria en España requiere que algunos contenidos, que tradicionalmente aparecen asociados exclusivamente a materias de lengua castellana y literatura, se incorporen a las mal llamadas áreas no lingüísticas.

La adquisición de la competencia ortográfica (entendiendo ortografía como parte de la expresión escrita y no como un apartado de la gramática) solo es posible integrada en todas las materias en las que se escribe; el uso compartido de vocabularios expresivos escritos (inventarios cacográficos) sería un recurso muy eficaz compartido por las áreas no lingüísticas, que obligaría a superar el enfoque tradicional sobre reglas y ejercicios ortográficos, 
claramente ineficaz en lengua española. Del mismo modo, es necesario unificar los textos que generan las diferentes habilidades cognitivolingüísticas (Jorba, 2000) según las necesidades de las diferentes áreas y la diversidad de cada hablante; describir, definir, resumir, explicar, justificar, argumentar y demostrar serían los ejes de la actividad lingüística en lengua española con los contenidos que aporta el currículo. La comprensión lectora de textos (académicos o no) relacionados con las ciencias sociales, científicos, tecnológicos y artísticos, sería el otro ámbito para integrar las competencias de las que tradicionalmente se ocupan las materias de lengua castellana y literatura en los contenidos de las áreas no lingüísticas.

El objetivo para el futuro es la generalización de la metodología característica de la enseñanza bilingüe en sus dos aspectos más significativos (el currículo integrado de las lenguas y el aprendizaje integrado de contenidos no lingüísticos y lenguas) en los proyectos lingüísticos de todos los centros; lo que implicará una renovación profunda de la práctica didáctica de la lengua de instrucción según el modelo que se aplica para la enseñanza de las otras lenguas. La competencia básica en comunicación lingüística, característica de la enseñanza obligatoria, constituirá de esta manera el eje fundamental de las materias de lengua castellana y literatura, superando una enseñanza de tradición lingüística y filológica poco eficiente en el siglo XXI.

\section{REFERENCIAS BIBLIOGRÁFICAS}

AA.VV. (2011). Orientaciones metodológicas para la elaboración y puesta en marcha de un proyecto lingüístico en los centros de la comunidad autónoma de Andalucía, Sevilla: Consejería de Educación [en línea], disponible en: http://www.juntadeandalucia.es/educacion/webportal/documents/21823/de81278d-a9f1-4d94-9608-6db222143b08, acceso el 23 de abril de 2012.

Bernárdez, E. (2008). El lenguaje como cultura. Madrid: Ariel.

Cassany, D., Luna, M. y Sanz, G. (1994). Enseñar lengua. Barcelona: Graó.

Cassany, D., Esteve, O. y Pérez-Vidal, C. (2009). El Porfolio Europeo de las lenguas en España (PEL), en Lenguaje y Textos, 29: 63-74.

Ganga, A. (2011). El Portafolio como recurso para la reflexión y la autoevaluación en alumnos con dificultades de aprendizaje, en Porta Linguarum, 16: 137-153.

Gómez Camacho, A. (2006). Los inventarios cacográficos en la enseñanza de la ortografía, en Escuela Abierta, 9: 63-74.

Gómez Camacho, A. (2007). Maldita tilde (Nuevos enfoques para la enseñanza de la ortografía en la educación secundaria y superior). Córdoba: Ediciones Toro Mítico.

Gómez Camacho, A. (2008). La educación secundaria de Andalucía y la ortografía del español, en Perspectiva CEP, 13: 109-132.

Gómez Camacho, A. (2009). La ortografía en la educación secundaria de Andalucía: vocabulario y enfoque didáctico, en Actas de las I jornadas de innovación e investigación educativa, Granada: Consejería de Educación, Junta de Andalucía.

Gómez Vidal, A. y Arcos, D. (2007). Plan de mejora de la competencia lingüística. Elaboración del Proyecto Lingüístico de Centro, en Avances en Supervisión Educativa, 7 [en línea], disponible en: http://adide.org/revista/index.php?option=com_content\&task=view\&id=224\& Itemid=49, acceso el 23 de abril de 2012.

Guillén, C. (2009). La concepción y la organización del currículo de lenguas en el sistema educativo español y el plurilingüismo como competencia, en Lenguaje y textos, 29: 47-62. 
Jorba, J., Gómez, I., Prat, A. (2000). Hablar y escribir para aprender. Uso de la lengua en situación de enseñanza-aprendizaje desde las áreas curriculares. Barcelona: Síntesis.

Junta de Andalucía (2011). El proyecto lingüístico de centro. Sevilla: Consejería de Educación [en línea], disponible en: http://www.juntadeandalucia.es/educacion/webportal/web/proyectolinguistico-centro/, acceso el 23 de abril de 2012.

Junyent, C. (coord.) (2007). Conclusions de la comissió Projecte lingüistic de centre. Per un projecte lingüistic per a l'escola catalana del segle XXI, [en línea], disponible en:

http://www20.gencat.cat/docs/Educacio/Documents/ARXIUS/consell-comissio_projecte.pdf, acceso el 21 de abril de 2012.

Lorenzo, F., Trujillo, F. y Vez, J. (2011). Educación bilingüe. Integración de contenidos y segundas lenguas. Madrid: Síntesis.

Lorenzo, F. (2010). Lingüística de la comunicación: El currículo multilingüe de géneros textuales, en Signos, 43(74), 391-410.

Lorenzo, F. , Casal, S. y Moore, P. (2009). The Effects of Content and Language Integrated Learning in European Education: Key Findings from the Andalusian Bilingual Sections Evaluation Projecten Applied Linguistics, 31/3: 418-442.

Lorenzo, F. (2007). The Sociolinguistics of CLIL: language Planning and Language Change in 21th Century Europe, en Revista Española de Lingüistica Aplicada, Vol. Extra 1: 27-38.

Madrid, D. y Hughes, S. (eds.) (2011). Studies in Bilingual Education. Bern: Peter Lang. Miret, I. y Ruiz Bikandi, U. (1997). El proyecto lingüístico de centro, en Textos, 13: 5-7.

Navarro, M. (2010). Competencias en el marco de didáctica de la lengua y la literatura, en Lenguaje y Textos, 32: 81-90.

Pavón, V. (2011). Principios teóricos y prácticos para la implantación de un modelo AICLE, en e-CO Revista digital de educación y formación del profesorado [en línea], disponible en $\mathrm{http}: / /$ revistaeco.cepcordoba.org/index.php?option $=$ com content\&view=article\&id=95: principios-teoricos-y-practicos-para-la-implantacion-de-un-modelo-aicle\&catid=12:monografico \&Itemid=58, acceso el 23 de abril de 2012.

Pérez Esteve, P. y Zayas, F. (2007). Competencia en comunicación lingüistica, Madrid: Alianza Editorial.

Ruiz, T. (2008). El tratamiento integrado de las lenguas. Construir una programación conjunta, en Textos, 47: 46-58.

Ruiz, U. (1997). Decisiones necesarias para la elaboracion del proyecto linguistico de centro, en Textos, 13, 9-24.

Sierra, J.M. y Lasagabaster, D. (2008). Los programas AICLE en aulas diversas: ¿una alternativa para todos?en Lenguaje y textos, 28: 131-142.

Trujillo, F. (2010). La competencia en comunicación lingüística como Proyecto de Centro: retos, posibilidades y ejemplificaciones, en Lenguaje y textos, 32: 35-40.

Trujillo, F. (2007). Enseñar nuevas lenguas en la escuela: L1, L2, LE..., NL, en Revista de Educación, 343: 71-91.

Vez, J. M. (2000). Fundamentos Lingüísticos en la enseñanza de las lenguas. Barcelona: Ariel.

Vollmer, H. J. (2007). Language and communication in the learning and teaching of science in secondary schools. Language Policy Division, Council of Europe, [en línea], disponible en: http://www.coe.int/t/dg4/Linguistic/Source/Prague07_LangCom_VollmerEd_EN.doc, acceso el 10 de abril de 2012. 